(1)

George Fox

UNIVERSITY
Digital Commons @ George Fox University

Faculty Publications - School of Physical

Therapy

School of Physical Therapy

2013

Total and Distributed Plantar Loading in Subjects With Stage II Tibialis Posterior Tendon Dysfunction During Terminal Stance

Christopher Neville

A. Samuel Flemister

Jeff Houck

Follow this and additional works at: https://digitalcommons.georgefox.edu/pt_fac

Part of the Physical Therapy Commons 


\title{
Total and Distributed Plantar Loading in Subjects With Stage II Tibialis Posterior Tendon Dysfunction During Terminal Stance
}

\author{
Christopher Neville, PhD, PT', A. Samuel Flemister, MD², and \\ Jeff Houck, PhD, PT $^{3}$
}

\begin{abstract}
Background: In subjects with stage II tibialis posterior tendon dysfunction (TPTD), the function of the tibialis posterior muscle is altered and may be associated with a change in total and distributed loading.

Methods: Thirty subjects with a diagnosis of stage II TPTD and I 5 matched control subjects volunteered to participate in a study to examine the total and distributed plantar loading under the foot during the terminal stance phase of gait. Plantar loading, measured as the subject walked barefoot, was assessed using instrumented flexible insoles. A secondary analysis was done to explore the contribution of flatfoot kinematics to plantar loading patterns.

Results: Overall, there was reduced total plantar loading in subjects with stage IITPTD compared with controls. Accounting for differences in total loading, the presence of clinically measured weakness in subjects with TPTD was associated with reduced lateral forefoot loading. Medial longitudinal arch height was significantly correlated with loading patterns but explained only $21 \%$ of the variance in observed loading patterns.

Conclusion: Subjects with TPTD who are strong exhibited loading patterns similar to controls. Changes in total and distributed loading during terminal stance suggest there are altered ankle mechanics at push-off during the functional task of gait.

Clinical Relevance: Strength, in the presence of TPTD, may be important to stabilize the midfoot during gait and might be important in rehabilitation protocols.
\end{abstract}

Keywords: gait, foot, kinetics, strength

Tibialis posterior tendon dysfunction (TPTD) has been cited as a leading cause of adult acquired flatfoot deformity leading to disability from altered gait patterns. ${ }^{1,17,34}$ The key features of the dysfunction include flatfoot deformity and tibialis posterior weakness. Dynamically, these features may be most apparent during the terminal stance phase of gait, which begins with the heel rising off the floor and ends when the opposite foot contacts the floor. During terminal stance, vertical load increases to exceed body weight as muscle forces are used to push forward onto the opposite leg. ${ }^{24}$ Simultaneously, the support surface progressively decreases as the heel comes off the floor and the foot is propelled over the metatarsal heads. This unique set of demands during terminal stance requires coordinated muscular efforts to progress over the foot in the anterior/posterior direction and to balance medial/lateral stability on the metatarsal heads. Plantar loading has been useful to investigate the effects of foot posture (flatfoot deformity) and to provide quantitative assessment of muscle dysfunction that may be useful in studying pathology of the tibialis posterior muscle. ${ }^{11}$

The tibialis posterior has primary roles of inversion at the subtalar joint and stabilization of the midfoot joints (calcaneocuboid, talonavicular, and midtarsal joints). ${ }^{18}$ Failure of the tibialis posterior tendon and its muscle weakness have been documented in subjects with stage II TPTD. ${ }^{12,36}$ The presence of abnormality (tendon degeneration and muscle weakness) may disrupt normal anterior/posterior and medial/lateral loading under the foot during terminal stance. ${ }^{16}$ The impact of TPTD on plantar loading has not been thoroughly investigated, but identifying altered loading patterns may help to target interventions focused on restoring normal foot movement and muscle function.

Weakness of the tibialis posterior may limit movement of the center of pressure anteriorly onto the metatarsal heads during gait, limiting the moment arm for the triceps surae to push off. Midfoot stability, aided by dynamic support from

\footnotetext{
'SUNY Upstate Medical University, Syracuse, NY

${ }^{2}$ University of Rochester Medical Center, Rochester, NY

${ }^{3}$ Ithaca College, Center for Foot and Ankle Research, Rochester, NY
}

\section{Corresponding Author:}

Christopher Neville, SUNY Upstate Medical University, Silverman Hall 2225, 750 E. Adams Street, Syracuse, NY 13210

Email: nevillec@upstate.edu 
the tibialis posterior, is necessary for progression of the body over the foot to the metatarsal heads during terminal stance. ${ }^{3,34}$ While one is walking, the force to move the center of mass forward to progress onto the metatarsal heads during terminal stance is largely a function of the triceps surae muscle group. ${ }^{24}$ Accompanying the triceps surae muscle force, the tibialis posterior is active to stabilize the midfoot. Failure to stabilize the midfoot with the tibialis posterior may limit the triceps surae from transmitting force from the heel to the metatarsal heads.

When the function of the tibialis posterior is simulated using a cadaver model, applying force through the tibialis posterior tendon results in shifting the center of pressure anteriorly. ${ }^{16}$ The anterior movement of the center of pressure indicates that vertical loading shifts toward the metatarsal heads and provides a greater moment arm for the triceps surae to act. Therefore, failure of the tibialis posterior may decrease the moment arm and total vertical plantar force produced by the triceps surae in the terminal stance phase.

Failure of the tibialis posterior tendon may lead to overloading of the medial side of the foot consistent with loss of inversion force and the development of the flatfoot deformity seen in stage II TPTD. ${ }^{1,17}$ During terminal stance, medial/lateral forces necessary to maintain a balanced load on the metatarsal heads are generated by the invertors (primarily the tibialis posterior) and evertors (primarily the peroneus longus and brevis). A cadaver experiment where the tibialis posterior was selectively unloaded demonstrated greater medial forefoot loading when tested in a terminal stance position. ${ }^{11}$ The opposite was true for unloading the peroneal muscles alone, which resulted in greater lateral forefoot loading. ${ }^{11}$ The unopposed force of the peroneal muscles in subjects with TPTD has been hypothesized to be a cause of the flatfoot deformity. ${ }^{21,35}$ Greater medial loading during terminal stance would be consistent with this theory.

Recent evidence suggests that not all subjects with stage II TPTD demonstrate weakness. ${ }^{26}$ A measure of clinical weakness in subjects with stage II TPTD was recently shown to be associated with abnormal foot kinematics and the presence of flatfoot deformity. ${ }^{26}$ Weakness may also be associated with alterations in plantar loading. Current theory suggests that the tibialis posterior contributes to medial/lateral movement of the subtalar joint and by stabilizing the midfoot may interact with the function of the triceps surae muscle in contributing to anterior/posterior movement of the center of pressure. These roles may be reflected in alterations in plantar loading in subjects with stage II TPTD.

The purpose of this study was to compare total and distributed loading patterns in subjects with stage II TPTD during the terminal stance phase of gait. We focused on terminal stance for 3 reasons: (1) It has been identified as a point of peak activity of the tibialis posterior muscle; (2) the peak vertical ground reaction force occurs at this point; and
(3) a large demand is placed on the foot with force transferred between the point of floor contact and the ankle while the heel is off the ground. ${ }^{11,28}$ Our primary hypothesis was that weakness associated with dysfunction of the tibialis posterior tendon would lead to a reduced total vertical load and an altered pattern of loading (increased medial loading, reduced lateral loading) when control subjects were compared with subjects with TPTD. Foot posture (flatfoot deformity) may also change plantar loading patterns, so a secondary purpose was to explore the effect of foot posture (flatfoot kinematics) on the pattern of foot loading in subjects with TPTD.

\section{Methods}

Thirty subjects with a diagnosis of stage II TPTD and 15 matched control subjects volunteered to participate in this study (Table 1). The control group was matched using body mass index and age due to their known effect on foot structure and gross function during walking. ${ }^{5,31}$ The control group was required to have a normal foot structure, defined using the arch height index and hindfoot measures in standing. All control subjects were required to have an arch height index greater than or equal to normal $(0.340)$ as reported by Butler et al. ${ }^{6}$ Additionally, goniometric measurements from a resting, standing position were taken after each subject was asked to stand in an alignment jig that positioned the feet $8.9 \mathrm{~cm}$ apart and toed out 7 degrees. All control subjects were required to have hindfoot positions relative to the leg that were between 2 degrees of inversion and 2 degrees of eversion. ${ }^{10}$ These measures ensured that control subjects would exhibit normal foot posture with theoretically balanced muscle forces for comparison to the group with TPTD.

The inclusion criteria for classification of stage II TPTD required subjects to have 1 or more signs related to tendinopathy, including (1) palpable tenderness of the tibialis posterior tendon, (2) swelling of the tibialis posterior tendon sheath, or (3) pain along the course of the tibialis posterior muscle or tendon while completing a single limb heel-rise, or inability to complete the heel-rise test. Additionally, 1 or more signs of flexible flatfoot deformity were required for classification of stage II TPTD. These included excessive nonfixed hindfoot eversion deformity during weight bearing, excessive forefoot abduction (too-many-toes sign), or demonstrated loss of height in the medial longitudinal arch. Signs of flatfoot deformity were based on comparisons from the involved to the uninvolved side. This then required that all subjects in the TPTD group have unilateral involvement. The uninvolved side may have also demonstrated signs of flatfoot deformity in some subjects but was not painful and did not demonstrate the same severity of flatfoot deformity (Table 1). Subjects were excluded if they had a 
Table I. Subject Classification Variables for Subjects With Stage IITPTD and Matched Controls ${ }^{\mathrm{a}}$

\begin{tabular}{|c|c|c|c|c|c|}
\hline & TPTD Total & TPTD Strong & TPTD Weak & Controls & $P$ Value \\
\hline Subjects, $n$ & 30 & 14 & 16 & 15 & \\
\hline Age, $y$ & $58.1 \pm 10.5$ & $57.9 \pm 11.4$ & $58.2 \pm 10.0$ & $56.5 \pm 7.7$ & .88 \\
\hline Height, cm & $167.2 \pm 8.7$ & $162.3 \pm 8.4$ & $|7| .6 \pm 6.5^{b, c}$ & $164.9 \pm 7.3$ & .004 \\
\hline Body mass, kg & $86.0 \pm 17.4$ & $80.2 \pm 14.6$ & $91.1 \pm 18.5$ & $83.2 \pm 10.8$ & .134 \\
\hline Body mass index & $30.6 \pm 5.4$ & $30.4 \pm 5.2$ & $30.8 \pm 5.7$ & $30.6 \pm 3.6$ & .98 \\
\hline Sex, n & $19 \mathrm{~F}, \mathrm{II} \mathrm{M}$ & $10 \mathrm{~F}, 4 \mathrm{M}$ & $9 \mathrm{~F}, 7 \mathrm{M}$ & I4 F, I M & $.06^{\mathrm{d}}$ \\
\hline $\mathrm{AHI}$ at $10 \%$ & $0.330 \pm 0.02$ & $0.34 \mathrm{I} \pm 0.02^{\mathrm{e}}$ & $0.321 \pm 0.02^{b, c}$ & $0.376 \pm 0.03$ & $<.001$ \\
\hline HF eversion involved & $9.8 \pm 4.2$ & $8.6 \pm 5.0^{\mathrm{e}}$ & $10.9 \pm 3.1^{\mathrm{b}}$ & $1.6 \pm 1.7$ & .03 \\
\hline HF eversion uninvolved & $8.9 \pm 4.5$ & $10.0 \pm 5.2^{\mathrm{e}}$ & $8.0 \pm 3.8^{b}$ & - & \\
\hline Duration of Symptoms, mo ${ }^{f}$ & & $11.0 \pm 12.1$ & $10.0 \pm 8.8$ & & .82 \\
\hline
\end{tabular}

Abbreviations: $\mathrm{AHI}$, arch height index; FF, forefoot; $\mathrm{HF}$, hindfoot; TPTD, tibialis posterior tendon dysfunction.

${ }^{a} V$ alues expressed as means \pm SD. $P$ values represent comparisons between TPTD groups and control group using a I-way analysis of variance.

${ }^{b}$ Denotes a significant difference (pairwise comparisons $P<.05$ ) between control and TPTD weak group.

'Denotes a significant difference (pairwise comparisons $P<.05$ ) between TPTD strong and TPTD weak group.

${ }^{\mathrm{d}}$ Results of Fisher exact statistical test to compare groups.

${ }^{e}$ Denotes a significant difference (pairwise comparisons $P<.05$ ) between control and TPTD strong group.

'Represents duration of reported symptoms at time of testing. 4 subjects ( 2 in each group) reported symptoms starting greater than 5 years ago but were unable to report a date. These subjects were not included in the data.

history of pain or abnormality in the foot or lower extremity that prevented them from ambulating greater than $15 \mathrm{~m}$. All subjects were required to have sensate feet to ensure their safety when walking. Subjects with other foot conditions, such as plantar fasciitis, were also excluded from the current study. All TPTD subjects were required to be at least 40 years of age to restrict the study to only those with the typical degenerative onset of TPTD. All subjects were informed of the experimental procedures and signed a consent form approved by the University of Rochester and Ithaca College research subject review boards.

\section{Isometric Test of Ankle Inversion and Foot Adduction Strength}

Deep posterior compartment strength was used as a primary independent variable to define groups; specifically, strength was used to divide the TPTD group into a weak and a strong group. A reliable and valid measure of deep posterior compartment strength is summarized below and also has been previously described. ${ }^{15,26}$ It was expected that TPTD subjects could be separated into 2 strength groups using a cutoff of $80 \%$ strength in the ratio of affected to unaffected side.

To measure deep posterior compartment muscle strength, data from a force transducer was viewed using an oscilloscope (TDS 410A, Tektronix, Beaverton, OR) as part of the strength setup to record and display maximum isometric force recorded from the deep posterior compartment. The oscilloscope visually displayed force from a maximum isometric ankle inversion and foot adduction strength effort. The force transducer (model SML-200, Interface, Scottsdale,
AZ) was connected in line with a resistance plate. The plate provided resistance to maximum efforts of ankle inversion and foot adduction. Padded supports fixed to the resistance plate allowed subjects to push from their resting foot position into forefoot adduction and ankle inversion. ${ }^{15}$

To assess the potential for the tibialis anterior muscle to mask the decrement in ankle inversion and foot adduction, electromyographic feedback was used. A surface electrode (DE-2.1, Delsys, Inc, Boston, MA) was placed over the skin of the tibialis anterior muscle. The surface electrode was connected to a 2-channel electromyography (EMG) system (Bangoli-2 EMG System, Delsys, Inc) for gain adjustments of 1 to $10 \mathrm{~K}$. An oscilloscope (TDS 410A, Tektronix) was used to visually display force and surface electromyography from the tibialis anterior muscle. The force and electromyography readings were taken directly from the oscilloscope. The digital display of the oscilloscope sampled data greater than $1000 \mathrm{~Hz}$.

\section{Procedures for Isometric Strength Testing}

Subjects were instructed to maintain plantar flexion force while performing a maximal voluntary ankle inversion and foot adduction effort. Subjects performed 5 to 7 practice submaximal efforts and 3 maximal efforts on the involved and uninvolved sides. Rest periods between maximal efforts were included to avoid fatigue (rest time 2-3 minutes as needed). Pain was rarely reported across the 30 subjects with TPTD tested in this study. This mirrored findings from the heel-rise test, which subjects were unable to do but rarely because of complaints of pain. Rather, subjects reported they just couldn't get their heel up despite trying. If the tibialis 
anterior EMG increased, subjects were instructed to push downward on the ball of their foot, providing a plantar flexor force to inhibit the tibialis anterior muscle. Prior to testing, a maximum voluntary effort in dorsiflexion against manual resistance was recorded. For the proposed study, peak force was normalized to body mass and averaged across the 3 maximal efforts. This procedure was repeated with the unaffected leg in order to calculate ratios between the affected and unaffected side. For the control group, a leg was randomly assigned at enrollment into the study to be the "involved" leg. The "involved" leg was used for the plantar loading measures and also was compared with the contralateral leg to calculate the strength ratio.

Following strength testing, 3 groups were defined for this study as follows: the control group with an average strength ratio of $1.06 \pm 0.1$ (range, 0.87-1.36), the TPTD strong group with an average ratio of $1.06 \pm 0.1$ (range, $0.89-1.25$ ), and the TPTD weak group with an average ratio of $0.64 \pm 0.2$ (range, $0.42-0.76$ ).

\section{Foot Loading}

Plantar loading, measured as the subject walked barefoot, was assessed using instrumented flexible insoles (Pedar-X, Novel Inc, St Paul, MN) secured to the plantar surface of each subject's foot with hypoallergenic tape. Each insole consisted of a matrix of 99 capacitive sensors located between 1-mm-thick protective coverings. Each sensor had a known area that varied based on the size of the insole. Prior to data collection, all insoles were calibrated according to manufacturer specifications using the TruBlu calibration device to read pressures in the $20-$ to $600-\mathrm{kPa}$ range. The Pedar system has been previously shown to be valid and reliable in measuring plantar pressures. ${ }^{19}$ Although designed for use in the shoe, the Pedar system is calibrated to accurately record pressure while walking barefoot and has been used in previous studies on floor surfaces ranging from carpet to concrete. ${ }^{22}$ For the purposes of this study, vertical loading under the foot was the primary interest, and thus data were analyzed converting pressure values to percentage of body weight. This conversion was done by dividing each of the summed pressure values for a given area of the insole by the summed area of the sensors contained in a mask. This value, in newtons, was then divided by each subject's weight in newtons. Thus, all values were newtons of plantar vertical force, divided by newtons of body weight, times 100. Subjects were fitted with an appropriate size pressure insole prior to attachment to the foot with a knee-high nylon stocking and adhesive skin tape. The information from the loading insoles was conveyed via telemetry to a remote computer as the subject walked. Data were collected at a 90-Hz sampling rate, consistent with previous studies, and to ensure that the pressure signal from the insole during the dynamic task of walking was captured without an aliasing error. ${ }^{29}$ A midgait method was used for analysis by eliminating the first 2 steps at the beginning and end of the gait trial to reduce the influence of speed fluctuations on plantar pressures at initiation and termination of gait. Subjects walked down a 10-m walkway at a walking speed constrained to be $1.0 \mathrm{~m} / \mathrm{s}$. This constraint was required to allow comparisons between groups. Average self-selected walking speed has been reported to be $1.4 \mathrm{~m} / \mathrm{s}$ with only minor changes $(<0.5$ $\mathrm{J} / \mathrm{kg} / \mathrm{m}$ ) in gross energy cost with speeds between 1.0 and $1.5 \mathrm{~m} / \mathrm{s}{ }^{5}$ This suggests that the dynamic function of the body to walk at speeds between 1.0 and $1.5 \mathrm{~m} / \mathrm{s}$ is consistent. It was expected that dynamic foot function would also be consistent at these speeds. This allowed comparisons between groups without the confounding effects of gait speed. During testing, speed was monitored using an infrared timing system (Brower, Salt Lake City, UT). A minimum of 5 steps were randomly identified and averaged. The peak pressure recorded across all trials and subjects did not exceed $407 \mathrm{kPa}$, suggesting that the insoles could accurately capture the pressure data based on calibration parameters.

Various regions of the foot (masks) were defined to describe the distributed plantar load on the pressure insole. The mask locations were the heel, midfoot, medial forefoot, lateral forefoot, medial toes, and lateral toes and were defined as a percentage of foot length and width but were checked for each subject by applying pressure to the insole with a blunt instrument under anatomic locations of interest. This check was done to ensure that variation in foot structure (such as the length of the first metatarsal) did not require a more custom mask than what would be predefined based on foot length. When necessary, subject-specific changes were made for each subject to correct these masks (Figure 1). The choice of mask locations was made to isolate the activity of muscles within the foot (flexor digitorum longus, lateral 4 toes; flexor hallucis longus, great toe) and to be consistent with previously reported literature. ${ }^{8,11}$ For the purposes of the current analysis, data for the heel and midfoot regions were combined (midfoot + heel) because of the typical heel-off position of the foot during terminal stance. Data were output as a percentage of body weight for comparison across masks and between groups.

For the current investigation, data were analyzed during late terminal stance corresponding to $75 \%$ of the stance phase of gait. The data for each subject were compared at this point in stance to allow comparisons across subjects, and between the control group and TPTD group, at a point where the functional demands on the foot and ankle were at a peak (second peak in the ground reaction force, and unique position of the foot during normal gait when the heel was off the floor). These demands would typically require peak activation of the tibialis posterior muscle and the gastroc soleus complex in the control group and served as a point of reference to determine the plantar loading occurring in the TPTD group. 


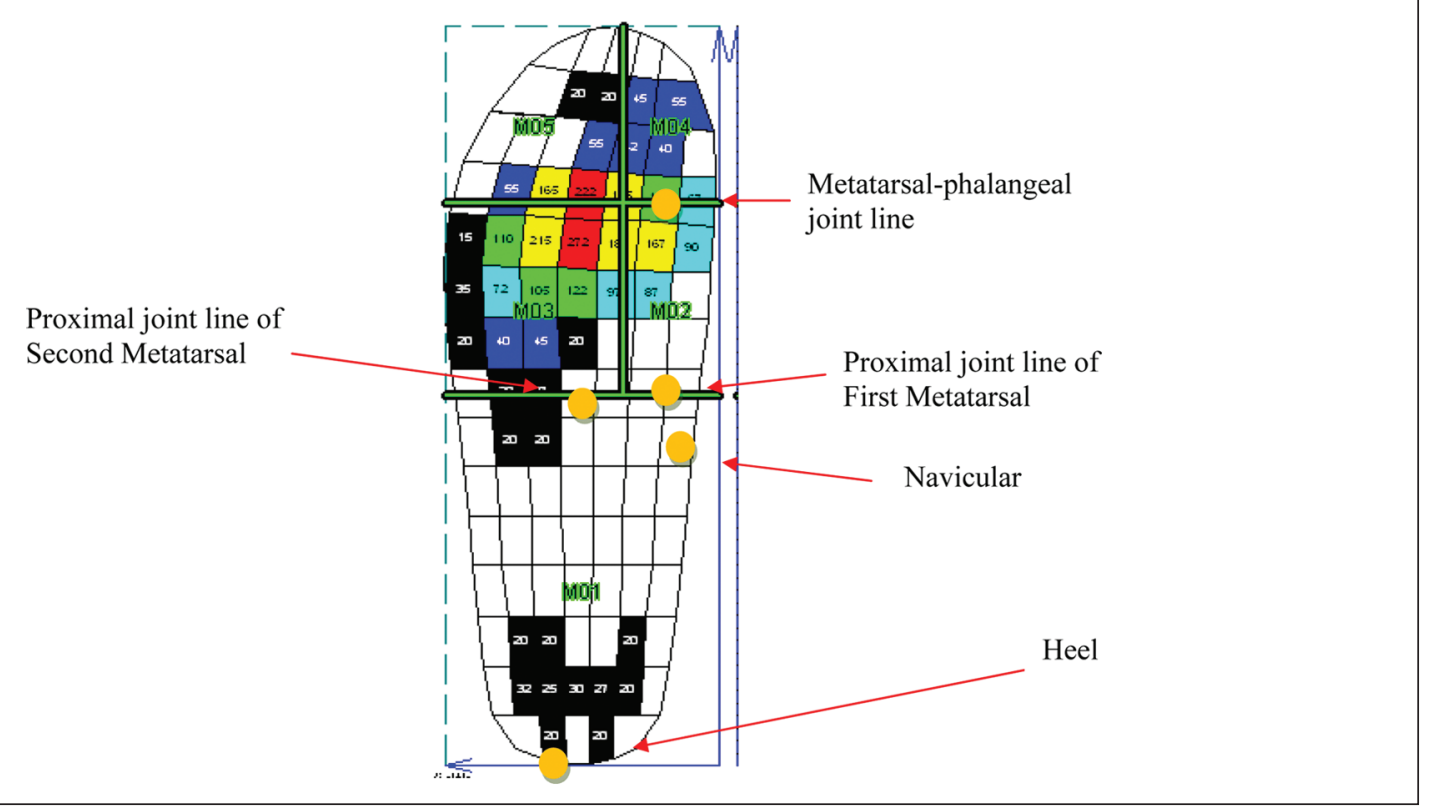

Figure I. Identification of plantar pressure masks (green lines) with subject-specific anatomic landmarks (palpation of bony landmarks identified by yellow dots) used to accurately identify loading areas specific to each subject. $\mathrm{MOI}=$ midfoot + heel, $\mathrm{MO} 2=$ medial forefoot, $\mathrm{MO} 3$ = lateral forefoot, $\mathrm{MO} 4=$ medial toes, $\mathrm{MO5}=$ lateral toes.

To address the primary hypothesis, a mixed effects 2-way analysis of variance was used with a between-subjects factor of group (3 levels; control, TPTD strong, TPTD weak) and a repeated factor of loading (6 levels; total, heel + midfoot, medial forefoot, lateral forefoot, medial toes, lateral toes). In the event that loading patterns were dependent on the group assignment, the main effects of loading were ignored and pairwise comparisons between the groups were explored maintaining an alpha level of .05 for all hypothesized comparisons. If a difference in total loading was observed between groups, then total loading was entered as a covariate for comparisons between loading regions. To address the second purpose, a stepwise linear regression was used to determine the kinematic factors that contributed to plantar loading at the terminal stance phase. Predictor variables included kinematic variables of flatfoot deformity including hindfoot inversion/eversion, forefoot abduction/ adduction, and medial longitudinal arch (MLA) height, which were collected as part of a previously reported study. ${ }^{26}$ These measures were included in this study to answer the question of how much flatfoot deformity contributed to changes in plantar loading in addition to the strength measures inherent to group assignment.

\section{Results}

The amount of total loading was found to be dependent on group (significant group $\times$ loading interaction, $P<.001$ ).
To explore the differences in total loading between groups, main effects were ignored and pairwise comparisons were explored between groups as proposed a priori.

The TPTD strong and TPTD weak groups demonstrated less total loading than the control group $(P=.016$ and $P=$ .001 , respectively) at the peak push-off point during terminal stance (Table 2 and Figure 2). There was no difference in total loading between the TPTD strong and TPTD weak groups $(P=.3)$. Due to the difference in total loading between groups, total loading was entered as a covariate and significantly $(P=.004)$ interacted with the comparisons between loading areas. Therefore, all comparisons between loading areas were done with total loading entered as a covariate. This allowed direct comparisons between the loading areas accounting for the differences in total loading between groups.

The weak TPTD group demonstrated significantly less lateral forefoot loading compared with the control group $(P<$ .001) during terminal stance. Additionally, the weak TPTD group demonstrated less $(P=.004)$ lateral forefoot loading compared with the strong TPTD group. No difference was observed between the TPTD strong group and the control group in lateral forefoot loading $(P=.16)$. No differences were observed in the medial or lateral toe regions or in the medial forefoot region between groups.

The TPTD weak group demonstrated greater midfoot + heel loading compared with the control group $(8.9 ; 95 \%$ confidence interval $[\mathrm{CI}], 20.1$ to $-2.1 ; P=.1$ ), but this was 
Table 2. Means (\% body weight) and SD of Loading in Areas of the Plantar Foot at the Terminal Stance Phase of Gait (75\%)

\begin{tabular}{lrcc}
\hline & \multicolumn{3}{c}{ Group Assignment } \\
\cline { 2 - 4 } Loading Area & \multicolumn{1}{c}{ Control } & TPTD Strong & TPTD Weak \\
\hline Total & $105.5 \pm 9.0$ & $97.9 \pm 8.3^{\mathrm{a}}$ & $94.8 \pm 7.1^{\mathrm{b}}$ \\
Medial toes & $15.1 \pm 2.7$ & $15.2 \pm 7.1$ & $13.9 \pm 6.5$ \\
Lateral toes & $11.7 \pm 3.4$ & $11.9 \pm 4.6$ & $10.5 \pm 6.7$ \\
Lateral FF & $55.2 \pm 9.1$ & $45.9 \pm 10.4$ & $34.5 \pm 9.6^{\mathrm{b}, \mathrm{c}}$ \\
Medial FF & $18.1 \pm 5.4$ & $18.1 \pm 7.5$ & $19.1 \pm 9.6$ \\
MF + Heel & $5.3 \pm 2.4$ & $6.8 \pm 7.0$ & $16.8 \pm 21.2$ \\
\hline
\end{tabular}

Abbreviations: FF, forefoot; MF, midfoot; SD, standard deviation; TPTD, tibialis posterior tendon dysfunction.

${ }^{a}$ Denotes a significant difference (pairwise comparisons $P<.05$ ) between control and TPTD strong group. For all loading areas except the total,

"total" was entered as a covariate when comparing groups.

"Denotes a significant difference (pairwise comparisons $P<.05$ ) between Control and TPTD weak group. For all loading areas except the total, "total" was entered as a covariate when comparing groups

'Denotes a significant difference (pairwise comparisons $P<.05$ ) between TPTD strong and TPTD weak group. For all loading areas except the total, "total" was entered as a covariate when comparing groups

not significant at the $<.05$ level. Additionally, the weak TPTD group demonstrated greater midfoot + heel loading compared with the strong TPTD group $(9.3 ; 95 \% \mathrm{CI}, 19.3$ to $-0.7 ; P=.07)$, but this was not significant at the $<.05$ level. No difference was observed between the TPTD strong group and the control group in midfoot + heel loading $(P=.41)$.

For lateral forefoot loading, the kinematic variable, MLA angle, significantly $(P=.01)$ contributed to the variance in lateral forefoot loading with a Pearson $r=-0.46$ and an $r^{2}=0.21$. This result suggests that greater lateral forefoot loading was associated with a higher MLA (smaller MLA angle). A significant correlation was also observed between hindfoot eversion and lateral forefoot loading $(P=.03)$, but this correlation did not contribute further to the regression model.

For combined loading under the midfoot and heel (midfoot + heel variable), the kinematic variable, MLA angle, significantly $(P=.03)$ contributed to the variance in midfoot + heel loading with a Pearson $r=-0.40$ and an $r^{2}=$ 0.16 . This result suggests that greater midfoot + heel loading was associated with a lower MLA (larger MLA angle). A significant $(P=.03)$ correlation was also observed between hindfoot eversion and midfoot + heel loading, but this correlation did not further contribute to the regression model.

\section{Discussion}

This study examined the total and distributed load on the foot during the terminal stance phase of gait when high loads are typically transmitted from the forefoot through the midfoot to the hindfoot. This period of stance requires coordinated muscular efforts to balance the large external dorsiflexion moment and prepare for ankle plantar flexion to push the body forward. ${ }^{11,14,24} \mathrm{New}$ to this study is evidence of altered total and distributed load in the presence of failure in a key extrinsic muscle, the tibialis posterior, associated with TPTD. The presence of weakness in subjects with TPTD influences this altered loading with greater reductions in lateral forefoot loading and a trend toward greater loading in the midfoot and heel. Flatfoot deformity contributes to plantar loading patterns in subjects with TPTD but explains a maximum of only $21 \%$ of the variance in loading, suggesting that strength (compared across groups) also contributes to altered plantar loading. Changes in total and distributed loading during terminal stance suggest altered ankle mechanics at push-off and unbalanced muscle forces to transfer force from the toes to the hindfoot.

A reduction in total terminal stance loading in subjects with stage II TPTD suggests modified force transmission through the foot by adopting compensatory mechanisms that result in unloading the foot, or weakness that alters force. Both pain and weakness have been linked to reductions in total loading during terminal stance. ${ }^{7,9,33}$ Studies of subjects with metatarsal or ankle arthritis report average $30 \%$ reductions in vertical loading during terminal stance likely due to unloading the painful foot area. ${ }^{27,32}$ When the effect of weakened inverters was tested, a reduced peak vertical load during terminal stance was theorized to be linked to failure of muscles such as the tibialis posterior to stabilize the midfoot. ${ }^{7}$ Additionally, reduced vertical loading was observed when testing the effects of tibial nerve blocks where the inverters and plantar flexors were inactive. ${ }^{33}$ In the current study, total vertical load was reduced on average between $8 \%$ and $11 \%$ compared with controls in both the weak and strong TPTD groups. The strong group may choose to unload the foot by flexing the hip as a compensatory mechanism to protect the tibialis tendon or other supporting ligaments. ${ }^{20}$ In the weak group, muscle weakness may contribute to altered total loading. ${ }^{23}$ In either group, these finding may suggest that postoperative rehabilitation may need to target restoring normal push-off gait patterns due to extended periods when compensation is likely.

The change in distributed load within the foot between controls and subjects with TPTD suggests an altered balance in the muscle forces used during terminal stance. The balanced contraction of the tibialis posterior as an inverter and the peroneus longus and brevis as evertors controls medial/lateral forefoot loading, whereas anterior/posterior loading is influenced by a controlled plantar flexion force from the triceps surae. ${ }^{2,21}$ Simulated loss of the tibialis posterior, tested in vitro, results in reduced lateral and greater medial forefoot loading as well as a shift toward more posterior loading in the foot. ${ }^{11,16}$ The current data are in agreement since the weak TPTD subjects demonstrated reduced 


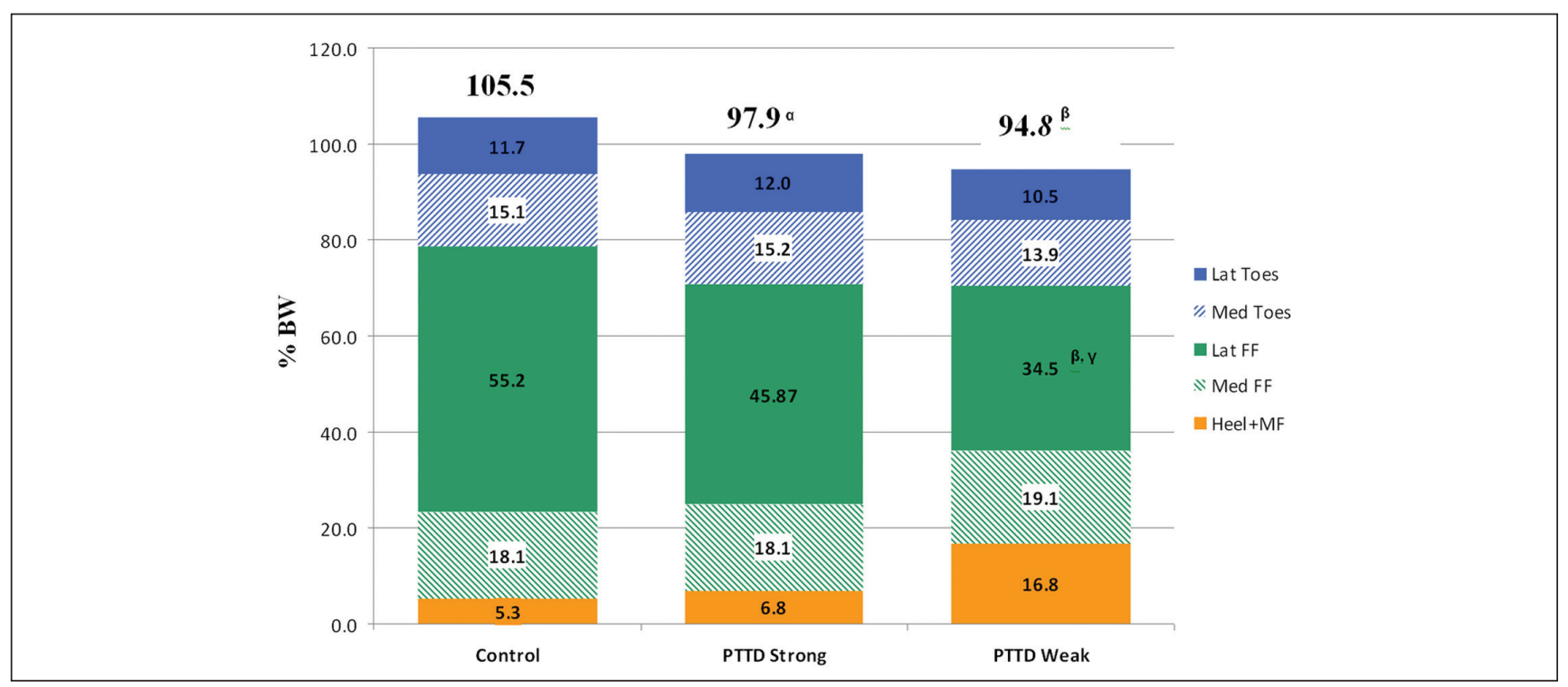

Figure 2. Mean total and distributed loading (\% body weight) by mask for 3 groups (TPTD strong, TPTD weak, and controls) at terminal stance (75\%).

Abbreviations: FF, forefoot; MF, midfoot; PTTD, posterior tibial tendon dysfunction

${ }^{\alpha}$ Denotes a significant difference (pairwise comparisons $P<0.05$ ) between Control and PTTD strong group.

${ }^{\beta}$ Denotes a significant difference (pairwise comparisons $P<0.05$ ) between Control and PTTD weak group.

${ }^{\gamma}$ Denotes a significant difference (pairwise comparisons $P<0.05$ ) between PTTD strong and PTTD weak group.

lateral forefoot loading. The current data diverge from data on the simulated role of the tibialis posterior with the subjects failing to increase medial forefoot loading during terminal stance. In vivo, subjects with TPTD who are strong may choose mechanisms that decrease the total load in terminal stance rather than increase medial forefoot loading. This may be a compensatory change to reduce the stress to medial stabilizing structures such as the tibialis posterior and spring ligament. The shift toward posterior (midfoot + heel) loading in the weak TPTD group was consistent with simulated tibialis posterior weakness but also with patient populations that experience Achilles tendon rupture. ${ }^{9}$ This is an interesting conclusion given that current operative procedures are used to target a short Achilles tendon in most patients with TPTD. Weakness of the tibialis posterior may reduce midfoot stability and interact with the ability of the triceps surae muscle group to effectively move plantar pressure more anterior into the forefoot.

The current study provides data on the change in total and distributed loading observed in subjects with TPTD with implications for how these changes may affect the task of walking. These findings should be considered noting the methods used to assign each group as well as the definition of masks to describe the areas of plantar loading. Haddad et $\mathrm{al}^{13}$ noted that stage II describes a continuum of deformity, and the definition of the stage continues to evolve. More recent descriptions of stage II include subclassification into types $\mathrm{A}, \mathrm{B}$, and $\mathrm{C}$ based on the observed forefoot deformity present. ${ }^{4}$ Additionally, the control group for this study consisted of mostly females to be consistent with previous samples of subjects with TPTD being greater than $80 \%$ female. ${ }^{1,25,30}$ However, the current sample of TPTD subjects was less female dominant, with 19 of the 30 volunteers $(63 \%)$ female and the subgroups even more gender equal (TPTD weak group, 9 of 16 female, or 56\%). This may suggest that future studies should consider the impact of gender on the severity of the condition or prevalence of weakness in subjects who are male. The current sample suggests that weakness may be more likely in males, with 7 of the 11 males in the weak group. The influence of ankle and foot range of motion (passive ankle dorsiflexion) in addition to the kinematics recorded in this study may further contribute to loading patterns but was not included in this study. These factors likely influence the reported results, whereas alternative choices for group assignment or loading masks may yield different results. Finally, this study focused on an isolated point $(75 \%)$ in the gait cycle to characterize the differences that exist in loading patterns due to muscle control and foot structure across groups at the end of the stance phase of gait. This study is cross-sectional in design and meant to provide insight for further theory generation to explain the link between loading patterns and muscle and tendon weakness in subjects with TPTD. Any reference to cause-and-effect relationships should be considered hypothetical and requires further research to determine usefulness in the clinical management of patients with TPTD. 
In conclusion, altered total and distributed loading in subjects with TPTD during the end of stance suggests impaired ability to achieve normal push-off mechanics. A decrease in total loading in all subjects with stage II TPTD suggests that alternative mechanisms, such as greater use of hip musculature, may be necessary to maintain forward progression. ${ }^{20}$ Additionally, altered distributed loading that is further influenced by weakness may place greater demands on medial structures such as the tibialis posterior tendon. Flatfoot posture may also contribute to plantar loading patterns in subjects with TPTD but explains a maximum of $21 \%$ of the variance in loading patterns.

\section{Declaration of Conflicting Interests}

The author(s) declared no potential conflicts of interest with respect to the research, authorship, and/or publication of this article.

\section{Funding}

Although none of the authors has received or will receive benefits for personal or professional use from a commercial party related directly or indirectly to the subject of this article, funds were received from Grant Number R15AR054507 from the National Institute of Arthritis And Musculoskeletal And Skin Diseases in support of the research or clinical study presented in this article.

\section{References}

1. Alvarez RG, Marini A, Schmitt C, Saltzman CL. Stage I and II posterior tibial tendon dysfunction treated by a structured nonoperative management protocol: an orthosis and exercise program. Foot Ankle Int. 2006;27:2-8.

2. Aronow MS, Diaz-Doran V, Sullivan RJ, Adams DJ. The effect of triceps surae contracture force on plantar foot pressure distribution. Foot Ankle Int. 2006;27:43-52.

3. Blackwood CB, Yuen TJ, Sangeorzan BJ, Ledoux WR. The midtarsal joint locking mechanism. Foot Ankle Int. 2005; 26:1074-1080.

4. Bluman EM, Title CI, Myerson MS. Posterior tibial tendon rupture: a refined classification system. Foot Ankle Clin. 2007; 12:233.

5. Browning RC, Baker EA, Herron JA, Kram R. Effects of obesity and sex on the energetic cost and preferred speed of walking. J Appl Physiol. 2006;100:390-398.

6. Butler RJ, Hillstrom H, Song J, et al. Arch height index measurement system: establishment of reliability and normative values. J Am Podiatr Med Assoc. 2008;98:102-106.

7. Christina KA, White SC, Gilchrist LA. Effect of localized muscle fatigue on vertical ground reaction forces and ankle joint motion during running. Hum Mov Sci. 2001;20:257-276.

8. Chuckpaiwong B, Nunley JA, Mall NA, Queen RM. The effect of foot type on in-shoe plantar pressure during walking and running. Gait Posture. 2008;28:405-411.
9. Costa ML, Kay D, Donell ST. Gait abnormalities following rupture of the tendo Achillis: a pedobarographic assessment. J Bone Joint Surg Br. 2005;87:1085-1088.

10. Diamond JE, Mueller MJ, Delitto A, Sinacore DR. Reliability of a diabetic foot evaluation [published correction appears in Phys Ther. 1989;69(11):994]. Phys Ther. 1989;69:797-802.

11. Ferris L, Sharkey NA, Smith TS, Matthews DK. Influence of extrinsic plantar flexors on forefoot loading during heel rise. Foot Ankle Int. 1995;16:464-473.

12. Goncalves-Neto J, Witzel SS, Teodoro WR, et al. Changes in collagen matrix composition in human posterior tibial tendon dysfunction. Joint Bone Spine. 2002;69:189-194.

13. Haddad SL, Myerson MS, Younger A, et al. Symposium: adult acquired flatfoot deformity. Foot Ankle Int. 2011;32:95-111.

14. Hamel AJ, Donahue SW, Sharkey NA. Contributions of active and passive toe flexion to forefoot loading. Clin Orthop Rel Res. 2001;393:326-334.

15. Houck JR, Nomides C, Neville CG, Samuel Flemister A. The effect of stage II posterior tibial tendon dysfunction on deep compartment muscle strength: a new strength test. Foot Ankle Int. 2008;29:895-902.

16. Imhauser CW, Siegler S, Abidi NA, Frankel DZ. The effect of posterior tibialis tendon dysfunction on the plantar pressure characteristics and the kinematics of the arch and the hindfoot. Clin Biomech. 2004;19:161-169.

17. Johnson KA, Strom DE. Tibialis posterior tendon dysfunction. Clin Orthop Rel Res. 1989;(239)196-206.

18. Kaye RA, Jahss MH. Tibialis posterior: a review of anatomy and biomechanics in relation to support of the medial longitudinal arch. Foot Ankle. 1991;11:244-247.

19. Kernozek TW, LaMott EE, Dancisak MJ. Reliability of an inshoe pressure measurement system during treadmill walking. Foot Ankle Int. 1996;17:204-209.

20. Lewis CL, Ferris DP. Walking with increased ankle pushoff decreases hip muscle moments. J Biomech. 2008;41:2082-2089.

21. Mizel MS, Temple HT, Scranton PE Jr, et al. Role of the peroneal tendons in the production of the deformed foot with posterior tibial tendon deficiency. Foot Ankle Int. 1999; 20:285-289.

22. Mohamed O, Cerny K, Jones W, Burnfield JM. The effect of terrain on foot pressures during walking. Foot Ankle Int. 2005;26:859-869.

23. Mueller MJ, Sinacore DR, Hoogstrate S, Daly L. Hip and ankle walking strategies: effect on peak plantar pressures and implications for neuropathic ulceration. Arch Phys Med Rehabil. 1994;75:1196-1200.

24. Neptune RR, Kautz SA, Zajac FE. Contributions of the individual ankle plantar flexors to support, forward progression and swing initiation during walking. J Biomech. 2001;34:1387-1398.

25. Ness ME, Long J, Marks R, Harris GF. Foot and ankle kinematics in subjects with posterior tibial tendon dysfunction. Gait Posture. 2008;27:331-339. 
26. Neville C, Flemister AS, Houck JR. Deep posterior compartment strength and foot kinematics in subjects with stage II posterior tibial tendon dysfunction. Foot Ankle Int. 2010;31:320-328.

27. O'Connell PG, Lohmann Siegel K, Kepple TM, Stanhope SJ, Gerber LH. Forefoot deformity, pain, and mobility in rheumatoid and nonarthritic subjects. J Rheumatol. 1998;25:16811686.

28. Perry J, Burnfield JB. Gait Analysis. Normal and Pathologic Function. Thorofare, NJ: SLACK Incorporated, 2010.

29. Rao S, Baumhauer JF, Becica L, Nawoczenski DA. Shoe inserts alter plantar loading and function in patients with midfoot arthritis. J Orthop Sports Phys Ther. 2009;39:522-531.

30. Ringleb SI, Kavros SJ, Kotajarvi BR, et al. Changes in gait associated with acute stage II posterior tibial tendon dysfunction. Gait Posture. 2007;25:555-564.

31. Scott G, Menz HB, Newcombe L. Age-related differences in foot structure and function. Gait Posture. 2007;26:68-75.
32. Shih LY, Wu JJ, Lo WH. Changes in gait and maximum ankle torque in patients with ankle arthritis. Foot Ankle. 1993;14: 97-103.

33. Sutherland DH, Cooper L, Daniel D. The role of the ankle plantar flexors in normal walking. J Bone Joint Surg Am. 1980;62:354-363.

34. Tome J, Nawoczenski DA, Flemister A, Houck J. Comparison of foot kinematics between subjects with posterior tibialis tendon dysfunction and healthy controls. J Orthop Sports Phys Ther. 2006;36:635-644.

35. Vertullo CJ, Nunley JA. Acquired flatfoot deformity following posterior tibial tendon transfer for peroneal nerve injury: a case report. J Bone Joint Surg. 2002;84-A:12141217.

36. Wacker J, Calder JD, Engstrom CM, Saxby TS. MR morphometry of posterior tibialis muscle in adult acquired flat foot. Foot Ankle Int. 2003;24:354-357. 